\title{
Marcadores de inflamación sistémica en pacientes ex-fumadores con enfermedad pulmonar obstructiva crónica en etapa estable
}

\author{
ARTURO MORALES S. ${ }^{1}$, JORGE DREYSE D. ${ }^{2}$, ORLANDO DÍAZ P. ${ }^{1}$,

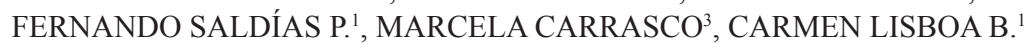

\section{Systemic inflammation among stable ex smokers with chronic obstructive pulmonary disease}

Background: Low-grade systemic inflammation is commonly observed in chronic obstructive pulmonary disease (COPD). Aim: To evaluate the extent of systemic inflammation in a group of ex-smokers with COPD in stable condition and its relation with pulmonary function and clinical manifestations. Patients and Methods: We studied 104 ex-smokers aged $69 \pm 8$ years (62 males) with mild to very severe COPD and 52 healthy non-smoker subjects aged $66 \pm 11$ years (13 males) as control group. High sensitivity serum $C$ reactive protein (CRP), interleukin 6 (IL6), fibrinogen (F) and neutrophil count $(\mathrm{Nc})$ were measured. Forced expiratory volume in the first minute $\left(F E V_{1}\right)$, inspiratory capacity (IC), arterial blood gases, six minutes walking test, dyspnea and body mass index (BMI) were measured, calculating the BODE index. Health status was assessed using the Saint George Respiratory Questionnaire (SGRQ), the chronic respiratory questionnaire (CRQ), registering the number of acute exacerbations (AE) during the previous year and inhaled steroids's use. Systemic inflammation was considered present when levels of CRP or IL6 were above the percentile 95 of controls $(7.98 \mathrm{mg} / \mathrm{L}$ and $3.42 \mathrm{pg} / \mathrm{ml}$, respectively). Results: COPD patients had significantly higher CRP and IL6 levels than controls. Their F and Nc levels were within normal limits. Systemic inflammation was present in 56 patients, which had similar disease severity and frequency of inhaled steroid use, compared with patients without inflammation. Patients with systemic inflammation had more $A E$ in the previous year; lower inspiratory capacity, greater dyspnea during the six minutes walk test and worse SGRQ and CRQ scores. Conclusions: Low-grade systemic inflammation was found in 56 of 104 ex-smokers with COPD. This group showed a greater degree of lung hyperinflation, dyspnea on exercise and poor quality of life.

(Rev Med Chile 2010; 138: 957-964).

Key words: Pulmonary disease, chronic obstructive; Systemic inflammation.

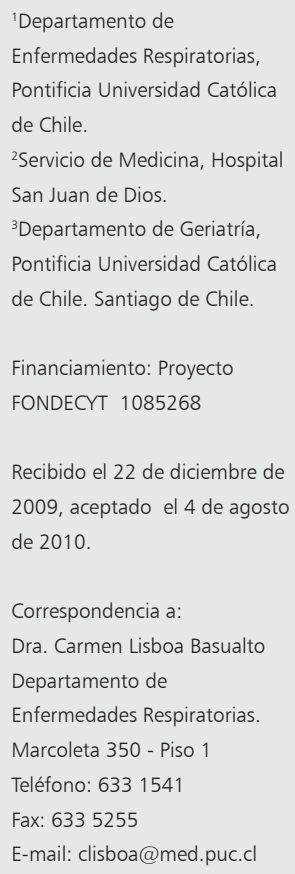

L a enfermedad pulmonar obstructiva crónica (EPOC) es una entidad caracterizada por Lestionion progresiva del flujo espiratorio que no es completamente reversible derivada de daño bronquiolar y enfisema pulmonar, asociada a una respuesta inflamatoria anormal del pulmón a la inhalación de gases y partículas nocivas ${ }^{1}$.
Últimamente se ha reconocido que la EPOC se asocia con frecuencia a algún grado de inflamación sistémica ${ }^{2}$. Es así como en pacientes en etapas estables de la enfermedad se ha descrito aumento de varios marcadores de inflamación sistémica en sangre periférica, tales como el factor de necrosis tumoral alfa (TNF- $\alpha$ ), interleuquinas (ILs), 
proteínas de fase aguda, fibrinógeno y recuento absoluto de neutrófilos ${ }^{2,3}$. A esta inflamación se ha atribuido algunos de los efectos sistémicos de la EPOC, como la pérdida de masa muscular, caquexia, osteoporosis y anemia entre otros ${ }^{4,5}$. Si bien no se conoce los mecanismos responsables de la inflamación sistémica, se ha planteado que esta sería mayor mientras más avanzada es la enfermedad pulmonar ${ }^{6}$. Otros factores asociados a inflamación sistémica pueden estar presentes en los pacientes con EPOC y contribuir así a esta condición, tales como la edad avanzada, tabaquismo, obesidad y enfermedad cardiovascular ${ }^{6,7}$. Si bien se sabe que el consumo de tabaco induce inflamación sistémica, el efecto de ser fumador activo o ex-fumador en los pacientes con EPOC ha sido escasamente evaluado ${ }^{7}$. Por otro lado, se ha descrito que el empleo de corticoides inhalados puede tener un efecto benéfico, reduciendo los niveles séricos de los marcadores inflamatorios ${ }^{7}$, aunque estos resultados no han sido confirmados.

La importancia clínica del aumento de los marcadores de inflamación sistémica en pacientes con EPOC reside en su asociación con el deterioro de la calidad de vida, riesgo de exacerbaciones ${ }^{8-10}$ y posiblemente con el pronóstico de la enfermedad ${ }^{11-14}$.

El objetivo de este estudio ha sido evaluar en un grupo de pacientes ex-fumadores, portadores de EPOC en etapa estable, los niveles de proteína $\mathrm{C}$ reactiva ultrasensible (PCRus), interleuquina 6 (IL6), fibrinógeno (F) y recuento absoluto de neutrófilos (RAN) séricos como marcadores de inflamación sistémica y su asociación con la gravedad clínica y funcional de la EPOC, la presencia de comorbilidades y el empleo de corticoides inhalados.

\section{Pacientes y Método}

\section{Pacientes}

Se estudiaron 104 pacientes ex-fumadores portadores de $\mathrm{EPOC}^{1}$, en condiciones estables de su enfermedad y que aceptaron participar en el protocolo firmando un acta de consentimiento informado aprobada por el comité de ética de nuestra institución. Estos pacientes pertenecen a una cohorte en seguimiento con el propósito de estudiar el efecto de las exacerbaciones sobre la progresión de la enfermedad. Los criterios de inclusión fueron ser mayores de 40 años, tener una historia de tabaquismo $\geq 10$ paquetes año, haber dejado de fumar por lo menos 6 meses antes, no haber presentado una exacerbación en el mes previo al ingreso y no poseer enfermedades que les impidiera realizar ejercicio físico o cuya gravedad no asegurara un seguimiento mínimo de 2 años. Se excluyó a los pacientes con historia de asma, bronquiectasias, secuelas de TBC, enfermedad neoplásica conocida o enfermedades caracterizadas por inflamación sistémica, como las enfermedades colágeno-vasculares.

\section{Controles}

Para establecer los valores de referencia de PCRus e IL6 séricas se estudiaron 52 individuos sanos no fumadores.

\section{Antecedentes clínicos}

Los pacientes se estudiaron en una etapa estable de la enfermedad, definida por la ausencia de exacerbaciones en las cuatro semanas previas. $\mathrm{Al}$ ingreso se registró las características demográficas, historia de tabaquismo, número de exacerbaciones en los últimos 12 meses, hospitalizaciones, comorbilidades y su tratamiento, y se midió la cotinina urinaria para comprobar su condición de no fumador.

\section{Evaluación de la inflamación sistémica}

Se midió la PCRus utilizando el método de inmunoensayo por nefelometría reforzado con partículas de látex (BN ProSpec equipment, DadeBehring ${ }^{\circledR}$, Deerfield, IL). La IL6 se determinó con el método de ELISA (Quantikine high sensitive ELISA R\&S Systems, Minneapolis). El fibrinógeno sérico se determinó con el método coagulométrico de Clauss. El RAN se realizó en un frotis sanguíneo con recuento celular diferencial.

Para el límite superior de lo normal de PCRus e IL6 sérica se empleó el promedio $\pm 2 \mathrm{DE}$ de los valores observados en el grupo de individuos sanos, los que fueron 7,98 mg/L y $3,42 \mathrm{pg} / \mathrm{ml}$ respectivamente. Para el fibrinógeno sérico fue 400 $\mathrm{mg} / \mathrm{dL}$ y para el recuento absoluto de neutrófilos fue sobre 7.000 células $/ \mathrm{mm}^{3}$.

\section{Evaluación clínica y funcional}

En todos los pacientes se evaluó la magnitud de la disnea utilizando la escala modificada del Medical Research Council del Reino Unido ${ }^{15}$ (MMRC). Se registró el número de exacerbaciones en los últimos 12 meses, el empleo de corticoides inhalados al momento del ingreso y las comorbi- 
lidades ${ }^{16}$. Se midió el peso y la talla, y se calculó el índice de masa corporal (IMC).

Se efectuó una espirometría según las normas de las Sociedades Americana de Tórax (ATS) y Europea de Enfermedades Respiratorias (ERS) ${ }^{17}$, que incluyó la medición de la capacidad inspiratoria (CI). Para el VEF y CVF se emplearon los valores de referencia de Hankinson y cols ${ }^{18}$ y para la CI, los de Lisboa y cols ${ }^{19}$. Se midió gases en sangre arterial y se efectuó una prueba de caminata en 6 minutos de acuerdo a las normas de la ATS ${ }^{20}$. Para esta última prueba se emplearon los valores de referencia de Troosters y cols ${ }^{21}$. Durante la caminata se registró la $\mathrm{SpO}_{2}$ y frecuencia cardíaca con oxímetro de pulso (Nonin Medical Inc., Plymouth, Mn., EE.UU.). La disnea y fatigabilidad de las piernas se evaluaron empleando la escala psicofísica de Borg ${ }^{22}$. Se calculó además el índice de gravedad multidimensional $\mathrm{BODE}^{23}$, que incluye el IMC, $\mathrm{VEF}_{1}$, disnea y distancia recorrida en 6 minutos.

\section{Calidad de vida}

Se utilizaron dos cuestionarios específicos para evaluar el estado de salud en pacientes con enfermedades respiratorias crónicas: las versiones en español del cuestionario de Saint's George (Saint's George Respiratory Questionnaire, SGRQ) ${ }^{24}$; y del cuestionario de enfermedades respiratorias crónicas (Chronic Respiratory Questionnaire, CRQ) ${ }^{25}$.

\section{Análisis estadístico}

Los resultados se expresan como promedio \pm 1DE. Se empleó la prueba t de Student para muestras independientes para las comparaciones entre pacientes y controles y para establecer las posibles diferencias entre pacientes con y sin elevación de marcadores inflamatorios. Para este último análisis, se consideró que un paciente tenía inflamación sistémica si PCRus y/o IL6 estaban por sobre los niveles de corte de los controles.

\section{Resultados}

\section{Características de los pacientes y controles}

La Tabla 1 resume las características de los 104 pacientes estudiados y de los 52 controles. Los pa-

Tabla 1. Características generales de pacientes con enfermedad pulmonar obstructiva crónica

\begin{tabular}{|c|c|c|c|}
\hline Variable & $\begin{array}{c}\text { EPOC } \\
n=104\end{array}$ & $\begin{array}{l}\text { Controles } \\
n=52\end{array}$ & Valor $\mathbf{p}$ \\
\hline Mujeres / Hombres & $42 / 62$ & $38 / 13$ & 0,0001 \\
\hline Edad, años & $69,2 \pm 8$ & $66,1 \pm 11$ & 0,0038 \\
\hline $\mathrm{IMC}, \mathrm{kg} / \mathrm{m}^{2}$ & $26,3 \pm 3,8$ & $27,6 \pm 4,2$ & 0,085 \\
\hline Paquetes/año & $42 \pm 24$ & - & \\
\hline$N^{\circ}$ exacerbaciones último año & $1,67 \pm 1,5$ & - & \\
\hline $\mathrm{PaO}_{2}$ basal, $\mathrm{mmHg}$ & $71 \pm 10$ & $77 \pm 6$ & 0,006 \\
\hline $\mathrm{VEF}_{1}, \%$ teórico & $53 \pm 20$ & $113 \pm 21$ & $<0,0001$ \\
\hline $\mathrm{Cl}$, \% teórico & $77 \pm 24$ & $101 \pm 21$ & $<0,0001$ \\
\hline DR6M, metros & $406 \pm 100$ & $540 \pm 68$ & $<0,0001$ \\
\hline DR6M, \% teórico & $83 \pm 24$ & $96 \pm 9$ & $<0,0001$ \\
\hline \multicolumn{4}{|l|}{ GOLD, n (\%) } \\
\hline Etapa I & $9(9 \%)$ & - & \\
\hline Etapa II & $48(46 \%)$ & - & \\
\hline Etapa III & $32(31 \%)$ & - & \\
\hline Etapa IV & $15(14 \%)$ & - & \\
\hline BODE, puntos & $2,87 \pm 2,1$ & - & \\
\hline SGRQ, puntos & $49 \pm 19$ & - & \\
\hline CRQ, puntos & $90 \pm 28$ & - & \\
\hline Índice de Charlson, puntos & $3,6 \pm 1,6$ & - & \\
\hline
\end{tabular}

Cl: capacidad inspiratoria; DR6M: Distancia recorrida en 6 minutos; SGRQ: Saint's George Respiratory Questionnaire; CRQ: Chronic Respiratory Questionnaire. 
cientes con EPOC pertenecían mayoritariamente a las etapas moderada y grave de la enfermedad; sólo $9 \%$ tenía enfermedad leve y $14 \%$, muy grave. Los niveles de cotinina fueron muy bajos $(11,45$ $\pm 28,13 \mathrm{ng} / \mathrm{ml})$.

El puntaje de gravedad de acuerdo al índice BODE fue bajo. El puntaje de comorbilidades, medido con el índice de Charlson también fue bajo, siendo las comorbilidades más frecuentes hipertensión arterial (55\%), enfermedad cardiovascular (17\%), diabetes mellitus (9\%) y antecedentes de neoplasia (5\% próstata o mama). Sólo 24 pacientes (23\%) no presentaron comorbilidades de acuerdo a lo propuesto por Charlson ${ }^{16}$.

\section{Marcadores de inflamación sistémica en EPOC estable}

El valor promedio de PCRus en los pacientes fue más elevado que en los controles $(5,45 \pm 0,88$ $\mathrm{mg} / \mathrm{L}$ vs $3,24 \pm 0,38 \mathrm{mg} / \mathrm{L} ; \mathrm{p}=0,004)$, al igual que la IL6 sérica $(5,3 \pm 1,89 \mathrm{pg} / \mathrm{ml}$ vs $1,89 \pm 0,109 \mathrm{pg} / \mathrm{ml}$; $\mathrm{p}=0,0002)$. En cambio, el fibrinógeno $(340 \pm 69$ $\mathrm{mg} / \mathrm{dL})$ y el RAN $\left(5.184 \pm 2.050 / \mathrm{mm}^{3}\right)$ estuvieron en rangos normales.

Se observó una correlación positiva entre los niveles séricos de PCRus e IL6 $(\mathrm{r}=0,32 ; \mathrm{p}=$ $0,0004)$, y entre PCRus y fibrinógeno $(r=0,50 ; p=$ $0,0001)$. La IL6 sérica se relacionó con el fibrinógeno $(\mathrm{p}=0,043)$ y el RAN se correlacionó solamente con los niveles de fibrinógeno sérico $(\mathrm{p}=0,038)$.

\section{Diferencias clínicas y funcionales en relación a la condición de inflamación}

De acuerdo a los criterios empleados en este estudio, 51 pacientes se consideraron inflamados y 53 no inflamados. La Tabla 2 muestra los índices clínicos y funcionales de ambos grupos. La edad, comorbilidades, IMC, gravedad de la EPOC según etapas GOLD (Figura 1) y el índice BODE fueron similares en ambos grupos. Tampoco hubo dife-

Tabla 2. Características de los pacientes con EPOC según presencia o no de inflamación

\begin{tabular}{|c|c|c|c|}
\hline & $\begin{array}{c}\text { No Inflamado } \\
n=53\end{array}$ & $\begin{array}{c}\text { Inflamado } \\
n=51\end{array}$ & Valor $\mathbf{p}$ \\
\hline Mujeres / Hombres & $20 / 33$ & $24 / 27$ & NS \\
\hline Edad, años & $68,9 \pm 1,0$ & $69,5 \pm 1,2$ & NS \\
\hline $\mathrm{IMC}, \mathrm{kg} / \mathrm{m}^{2}$ & $25,8 \pm 0,5$ & $27,1 \pm 0,6$ & NS \\
\hline Paquetes/año & $46 \pm 3,1$ & $38 \pm 3,4$ & NS \\
\hline $\mathrm{N}^{\circ}$ exacerbaciones último año & $1,20 \pm 0,2$ & $2,14 \pm 0,2$ & 0,002 \\
\hline $\mathrm{PaO}_{2}, \mathrm{mmHg}$ & $72 \pm 1,5$ & $69 \pm 1,3$ & NS \\
\hline VEF $\%$ teórico & $53 \pm 2,9$ & $52 \pm 2,8$ & NS \\
\hline $\mathrm{Cl}$, \% teórico & $83 \pm 3,4$ & $73 \pm 2,8$ & 0,034 \\
\hline DR6M, metros & $414 \pm 12$ & $397 \pm 16$ & NS \\
\hline DR6M, \% teórico & $85 \pm 2,6$ & $85 \pm 3,4$ & NS \\
\hline $\begin{array}{l}\text { GOLD } \\
\text { Etapa I } \\
\text { Etapa II } \\
\text { Etapa III } \\
\text { Etapa IV }\end{array}$ & $\begin{array}{r}6 \\
23 \\
16 \\
8\end{array}$ & $\begin{array}{r}3 \\
25 \\
16 \\
7\end{array}$ & NS \\
\hline Fibrinógeno, mg/dL & $321 \pm 7$ & $360 \pm 11$ & 0,003 \\
\hline Neutrófilos, células $/ \mathrm{mm}^{3}$ & $4.762 \pm 185$ & $5.624 \pm 354$ & 0,031 \\
\hline Índice BODE, puntos & $2,8 \pm 0,3$ & $3,1 \pm 0,3$ & NS \\
\hline SGRQ, puntos & $45 \pm 2$ & $53 \pm 3$ & 0,023 \\
\hline CRQ, puntos & $100 \pm 3$ & $80 \pm 5$ & $<0,001$ \\
\hline Disnea post ejercicio, Borg & $4,12 \pm 0,3$ & $5,04 \pm 0,3$ & 0,046 \\
\hline
\end{tabular}

Cl: capacidad inspiratoria; DR6M: Distancia recorrida en 6 minutos; SGRQ: Saint's George Respiratory Questionnaire; CRQ: Chronic Respiratory Questionnaire. 


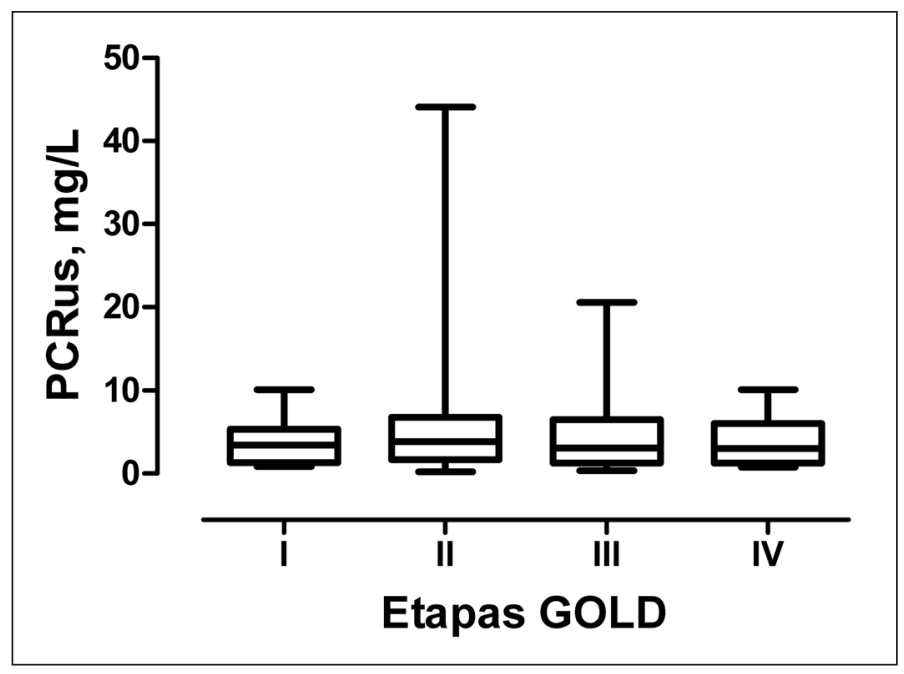

Figura 1. Se describe los valores de proteína $C$ reactiva ultrasensible (PCRus) en pacientes con enfermedad pulmonar obstructiva crónica, clasificada según categorías de GOLD. No se observó diferencias entre las distintas categorías. rencias en las vacunaciones recibidas, uso crónico de $\mathrm{O}_{2}$ o tratamiento con corticoides inhalatorios. En cambio, los pacientes con inflamación sistémica tuvieron más exacerbaciones durante el año previo, mayor hiperinflación pulmonar, mayor disnea al final de la prueba de caminata y peor calidad de vida. También presentaron valores significativamente más altos de F y RAN.

\section{Discusión}

Nuestros resultados demuestran que en pacientes ex-fumadores con EPOC, en etapa estable de la enfermedad, existe un estado de inflamación sistémica de bajo grado. Esta inflamación no guarda relación con la gravedad de la EPOC, evaluada según GOLD y BODE, y no es afectada por el empleo de corticoides inhalatorios. En cambio, es influida por las exacerbaciones, siendo mayor en aquellos pacientes con $\geq 2$ exacerbaciones el año previo. Por último, los pacientes que presentaron inflamación sistémica tenían mayor hiperinflación pulmonar y disnea al final del ejercicio, y una peor calidad de vida.

En el presente estudio la inflamación sistémica fue investigada midiendo algunos de los marcadores séricos más frecuentemente examinados en pacientes con EPOC ${ }^{3}$. Los valores de PCRus fueron más elevados que en el grupo control y semejantes a los comunicados en varios estudios realizados en condiciones estables de la $\mathrm{EPOC}^{7,26}$ y en pacientes con EPOC en el estudio epidemiológico de Framingham ${ }^{11}$, pero son más elevados a los comunicados por otros autores ${ }^{8,14}$. El aumento de IL6 sérica ha sido comunicado en menos estu$\operatorname{dios}^{10,11,27}$ y sus niveles en etapa estable han sido variables. Los de nuestros pacientes son similares a los comunicados por Walter y cols ${ }^{11}$. Los niveles de fibrinógeno estuvieron dentro del rango normal, al igual que el RAN, y difieren de lo observado en otros estudios $^{8,28}$.

Hasta ahora se desconoce el origen de la inflamación sistémica en la EPOC y la hipótesis que se deba a rebalse ("spill-over") de la inflamación pulmonar hacia la circulación sistémica no ha logrado ser confirmada. Hurst y cols ${ }^{27}$, por ejemplo, no observaron relación entre la magnitud de la inflamación en las vías aéreas y la respuesta inflamatoria sistémica. A su vez, Vernooy y asociados $^{29}$ tampoco encontraron relación entre los marcadores de inflamación en el esputo inducido y en la sangre. Recientemente, evaluamos durante una exacerbación la inflamación de las vías aéreas mediante medición de óxido nítrico exhalado y la magnitud de la inflamación sistémica con marcadores séricos y no observamos relación entre ellos ${ }^{30}$.

Como alternativas para explicar la inflamación sistémica de la EPOC se ha planteado el efecto inflamatorio del tabaquismo activo, aun cuando la inflamación persiste después del abandono del tabaquismo; y la hiperinflación pulmonar, a través de la estimulación y liberación de citoquinas 
desde el pulmón. También se ha postulado que puede influir la gravedad de la enfermedad ${ }^{6,11,28}$, la obesidad, las comorbilidades y la edad avanzada ${ }^{31}$. Y otra hipótesis sugiere que la EPOC podría ser una forma de expresión de un estado inflamatorio sistémico con compromiso de muchos órganos ${ }^{5,32}$. En relación a estos planteamientos, cabe destacar que nuestros pacientes con inflamación sistémica tenían hiperinflación pulmonar, objetivada por una disminución de la CI. La asociación entre niveles plasmáticos elevados de PCRus y reducción del $\mathrm{VEF}_{1}$, comunicada por varios autores ${ }^{6,11,28}$, no fue observada en nuestro estudio, ya que no hubo relación entre inflamación y gravedad de la EPOC según GOLD. Aun cuando se sabe que el tabaquismo por sí mismo puede inducir inflamación sistémica, poco se conoce respecto al efecto del tabaquismo activo o su suspensión sobre el curso de la inflamación en la EPOC. Según Gan y cols ${ }^{33}$ la presencia de inflamación es independiente de la condición de fumador activo o ex-fumador. Por utilizar la base de datos del estudio NANHES III, su comparación con el presente estudio es difícil pues la reducción del $\mathrm{VEF}_{1}$ no excluía a pacientes asmáticos y no se evaluaron las comorbilidades ni el efecto potencial de las exacerbaciones. Por su parte, Pinto-Plata y cols ${ }^{7}$ observaron que los niveles de PCR eran similares en pacientes ex-fumadores y fumadores activos con EPOC, pero en su muestra de 88 pacientes sólo 14\% eran fumadores activos. Los datos del presente estudio apoyan sus resultados, pues aun cuando no incluimos fumadores activos, confirmamos la presencia de inflamación en los ex-fumadores. Que los niveles de PCR no difieran entre fumadores activos y ex-fumadores sugiere que la presencia de inflamación puede representar un fenotipo de la EPOC. Si esto fuese así, la identificación de este fenotipo podría tener implicancias de valor pronóstico y terapéutico.

El efecto de los corticoides inhalados sobre la inflamación sistémica ha proporcionado resultados variables. Mientras que algunos estudios han observado que su empleo reduce los marcadores sistémicos de inflamación ${ }^{7,34}$, otros no han demostrado dichos efectos ${ }^{35,36}$, concordando con nuestros resultados.

La importancia de evaluar el aumento de los niveles de marcadores de inflamación sistémica en los pacientes con EPOC deriva en parte de su asociación y posible causalidad con otras enfermedades que se observan frecuentemente en esta población, como enfermedades cardiovasculares, osteoporosis, atrofia muscular, y obesidad, entre otras. Algunos autores han demostrado, además, que la elevación de algunos marcadores se asocia con mortalidad, riesgo de exacerbaciones y declinación acelerada de la enfermedad. La PCR como índice pronóstico de EPOC ha sido evaluada por Dahl y asociados ${ }^{12}$ en una cohorte de 1.300 sujetos durante un período de seguimiento de 8 años. La incidencia de hospitalizaciones por EPOC y de muertes fueron mayores en los individuos con una PCRus mayor de 3,0 mg/L, con un riesgo relativo de 1,4 y 2,2 para hospitalización y muerte, respectivamente, después de ajustar por edad, $\mathrm{VEF}_{1}$, género, cardiopatía coronaria y tabaquismo. Sin embargo, esta asociación con mortalidad no fue corroborada por de Torres y cols ${ }^{14}$ en un grupo de 218 pacientes con EPOC leve a muy grave seguidos por una mediana de 36 meses.

Las exacerbaciones de la enfermedad ocurren frecuentemente en los pacientes con EPOC, especialmente en las etapas más avanzadas. Se ha planteado que la inflamación sistémica podría ser un factor de riesgo de exacerbaciones. Así, Parera y cols ${ }^{10}$ demostraron que la persistencia de niveles elevados de PCR después de 14 días del inicio de una exacerbación es predictor de una nueva exacerbación en los siguientes dos meses. Los niveles elevados de fibrinógeno también parecen asociarse a un mayor riesgo de hospitalización ${ }^{28}$.

El rol de la inflamación sistémica en la reducción de la función pulmonar y/o en la progresión de la enfermedad ha sido investigado en cohortes seguidas por varios años con resultados contradictorios. ${ }^{12,13}$ Las alteraciones clínico funcionales observadas en los pacientes con inflamación sistémica del presente estudio concuerdan parcialmente con los resultados de Broekhuizen y cols ${ }^{6}$, quienes demostraron que la PCRus es un marcador de compromiso de la capacidad funcional y de la calidad de vida, independientemente del VEF $F_{1}$. En el presente estudio, no observamos una disminución de la capacidad para caminar, pero sí mayor hiperinflación pulmonar, disnea, y deterioro de la calidad de vida.

En resumen, alrededor de 50\% de los pacientes en etapa estable de este estudio presentaron aumento de PCRus y/o IL6, independientemente de la gravedad de la EPOC y del uso de corticoides inhalatorios. Estos pacientes se caracterizaron por haber presentado más exacerbaciones en el año 
previo, tener mayor hiperinflación pulmonar y una peor calidad de vida que aquellos no inflamados. La identificación de estos pacientes parece ser relevante en la medida que la inflamación sistémica se asocia al riesgo de exacerbaciones y probablemente a un mal pronóstico de la enfermedad. Sin embargo, para establecer su real importancia es necesario observar el comportamiento de estos pacientes en el largo plazo.

\section{Referencias}

1. Global initiative for the Chronic Obstructive Lung Disease. Global strategy for the diagnosis, management and prevention of COPD. December2008http://www. goldcopd.com/Guidelineitem.

2. Maninno DM, Ford ES, Redd SC. Obstructive and restrictive lung disease and markers of inflammation in chronic obstructive pulmonary disease: data from the third National and Nutrition examination. Am J Med 2003; 114: 758-62.

3. Gan WQ, Man SPF, Senthilselvan A, Sin DD. Association between chronic obstructive pulmonary disease and systemic inflammation: a systematic review and a metaanalysis. Thorax 2004; 59: 574-80.

4. Agusti AGN, Noguera A, Sauleda J, Sala E , Pons J. Busquets X. Systemic effects of chronic obstructive pulmonary disease. Eur Respir J 2003; 21: 347-60.

5. Barnes PJ, Celli BR. Systemic manifestations and comorbidities of COPD. Eur Respir J 2009; 33:1165-85.

6. Broekhuizen R, Wouters EFM, Creutzberg EC, Scholz AMWJ. Raised CRP levels mark metabolic and functional impairment in advanced COPD. Thorax 2006; 61: 17-22.

7. Pinto-Plata VM, Mullerova H, Toso JF, Feudjo-Tapie $\mathrm{M}$, Soriano JB, Vessey RS, et al. C- reactive protein in patients with COPD, control smokers and non-smokers. Thorax 2006; 61: 23-8.

8. Groenewegen KH, Postma DS, Wim C Hop J, Pascal LM, Wielders L, et al. Increased systemic inflammation is a risk factor for COPD exacerbations. Chest 2008; 133: 350-7.

9. Pinto-Plata V, Livnat G, Girish M, CabraL H, Masdin P, Linacre P, et al Systemic Cytokines, clinical and physiological changes in patients hospitalized for exacerbations Chest 2007; 131: 37-43.

10. Parera WR, Hurst JR, Wilkinson TMA, Sapsford RJ, Müllerova H, Donaldson GC, et al. Inflammatory changes, recovery and recurrence at COPD exacerbation. Eur Respir J 2007; 29: 527-34.

11. Walter RE, Wilk JM, Larson MG, Vasan RS, Keaney JF, lipinska I, et al. Systemic inflammation and COPD. The Framingham heart study. Chest 2008; 133: 19-25.

12. Dahl M, Vestbo J, LAnge P, Bojesen SE, Tybjaerg-Hansen A, Nordestgaard BG. C-reactive protein as a predictor of prognosis in chronic obstructive pulmonary disease. Am J Respir Crit Care Med 2007; 175: 250-5.

13. Fogarty AW, Jones S, Britton JR, Lewis S, McKeever TC. Systemic inflammation and decline in lung function in a general population: a prospective study. Thorax 2007; 62: 515-20.

14. De Torres JP, Pinto-Plata V, Casanova C, Múllerova H, Córdoba-Lanús E, Muros de Fuentes M, et al. C-reactive protein levels and survival in patients with moderate to very severe COPD. Chest 2008; 133: 1336-43.

15. Mahler DA, Wells CK. Evaluation of clinical methods for rating dyspnea. Chest 1988; 93: 580-6.

16. Charlson M, Szatrowski TP, Peterson J, Gold J. Validation of a combined comorbidity index. J Clin Epidemiol 1994; 47: 1245-51.

17. Miller MR, Hankinson J, Brusasco V, Burgos F, Casaburi $\mathrm{R}$, Coates A, et al. Standardisation of spirometry. Eur Respir J 2005; 26: 319-23.

18. Hankinson JL, Odencrantz JR, Fedan KB: Spirometric reference values from a sample of the general U.S. Population. Am J Respir Crit Care Med 1999; 159: 179-87.

19. Lisboa C, Leiva A; Pinochet R, Repetto P, Borzone G, Díaz O. Valores de referencia de la capacidad inspiratoria en sujetos sanos no fumadores mayores de 50 años. Arch Bronconeumol 2007; 43: 485-9.

20. American Thoracic Society Statement. Guidelines for the six-minute walk test. Am J Respir Crit Care Med 2002; 166: 111-7.

21. Troosters T, Gosselink S, Decramer M. Six minute walking distance in healthy elderly subjects. Eur Respir J 1999; 14: 270-4.

22. Borg GAV. Psychophysical basis of perceived exercise. Med Sci Sports Exerc 1982; 14: 337-81.

23. Celli BR, Cote CG, Marín JM, Casanova C, Montez de Oca M, Méndez RA, et al. The body-mass index, airflow obstruction, dyspnea and exercise capacity index in chronic obstructive pulmonary disease. N Engl J Med 2004; 350: 1005-12.

24. Jones PW, Quirk FH, Baveystock CM, Littlejohns P. A self complete measure for chronic airflow limitation: the St George's Respiratory Questionnaire. Am Rev Respir Dis 1992; 142: 1321-7.

25. Guyatt GH, Berman LM, Townsed M, Pugleys M, Chambers LW. A measured of quality of life for clinical trials in chronic lung disease. Thorax 1987; 47: 773-8.

26. Garrod R, Marshall J, Barley E, Fredericks S, Hagarr G. The relationship between inflammatory markers and 
Marcadores de inflamación sistémica en enfermedad pulmonar obstructiva crónica - A. Morales S. et al

disability in chronic obstructive pulmonary disease. Primary Care Respiratory Journal 2007;16: 236-40.

27. Hurst J R, Wilkinson TMA, Perera WR, Gavin C, Donaldson GC, Wedzicha JA. Relationships among bacteria, upper airway, lower airway and systemic inflammation in COPD. Chest 2005; 127: 1219-122.

28. Dahl M, Tybjaerg-Hansen A, Vestbo J, Lange P, Nordestgaard BC. Elevated plasma fibrinogen associated with reduced pulmonary function and increased risk of chronic obstructive pulmonary disease Am J Respir Crit Care Med 2001; 164: 1008-11.

29. Vernooy JH, Kucukaycan M, Jacobs JA, Chavannes NH, Buurman WA, Dentener MA, et al. Local and systemic inflammation in patients with chronic obstructive pulmonary disease: soluble tumor necrosis factor receptors are increased in sputum Am J Respir Crit Care Med 2006; 166: 1218-24.

30. Lisboa C, Dreyse J, Leiva A, Arancibia F, Saldías F, Díaz O. Oxido nítrico exhalado en condiciones estables y durante las exacerbaciones en pacientes con enfermedad pulmonar obstructiva crónica. Rev Chil Enf Respir
2009; 25: 259.

31. Agusti A. Systemic effects of chronic obstructive pulmonary disease. Proc Am Thorac Soc 2007; 4: 522-5.

32. Fabbri LM, Luppi F, Beghé B, Rabe KF. Complex chronic comorbidities of COPD. Eur Respir J 2008; 31: 204-12.

33. Gan WQ, Man SFP, Sin DD. The interactions between cigarette smoking and reduced lung function on systemic inflammation. Chest 2005; 127: 558-64.

34. Sin DD, Lacy P, York E, Man SFP. Effects of fluticasone on systemic markers of inflammation in chronic obstructive pulmonary disease. Am J Respir Crit Care Med 2004; 170: 760-5.

35. Sin DD, Man SFP, Marciniuk DD, Ford G, Fitz Gerald M, Wong E, et al. The effects of fluticasone with or without salmeterol on systemic biomarkers of inflammation in chronic obstructive pulmonary disease. Am J Respir Crit Care Med 2008; 177: 1207-14.

36. Perng D-W, Tao C-W, Su K-C, Tsai C-C, Liu L-Y, Lee Y-C. Anti-inflammatory effects of salmeterol/fluticasone, tiotropium/fluticasone or tiotropium in COPD. Eur Respir J 2009; 33: 778-84. 